\title{
Valutazione dell'inquinamento da rumore in una Unità di Terapia Intensiva Nefrologica e Dialisi
}

\author{
M. Buemi ${ }^{1}$, A. Allegra' ${ }^{2}$, F. Grasso ${ }^{3}$, G. Mondio ${ }^{4}$ \\ ${ }^{1}$ Dipartimento di Medicina Interna, Cattedra di Nefrologia II, \\ ${ }^{2}$ Cattedra di Patologia Medica I, Policlinico Universitario di Messina \\ ${ }^{3}$ Dipartimento di Statistica \\ ${ }^{4}$ Dipartimento di Fisica della Materia e Fisica dell'Ambiente \\ Centro Siciliano per le Ricerche Atmosferiche e di Fisica dell'ambiente - Messina
}

a polluzione sonora costituisce una delle forme più insidiose e probabilmente più sottovalutate di inquinamento ambientale. Il danno da rumore costituisce infatti una delle malattie professionali più frequenti e di maggiore aggravio economico per la società. Accanto agli effetti uditivi del rumore, sordità di tipo percettivo bilaterale e ad andamento progressivo, che interessa sia la trasmissione aerea che ossea, sono ormai noti gli effetti extrauditivi del rumore, ed il danno da rumore sembra poter coinvolgere tutti gli organi ed apparati con l'eccezione forse del sistema muscolo-scheletrico.

Sebbene in letteratura esistano segnalazioni discordanti, sembra assodato che il rumore possa modificare l'attività del sistema endocrino, dell'apparato digerente, del sistema nervoso centrale e soprattutto dell'apparato cardiovascolare. Vari Autori hanno infatti segnalato la possibilità che l'esposizione al rumore possa determinare un aumento della frequenza respiratoria, l'aumento dell'incidenza di ulcere gastriche e duodenali e di stati spastici del colon (1).

Per quanto riguarda il sistema endocrino il rumore sarebbe capace di influenzare l'asse ipofiso-surrenalico, determinando un aumento della secrezione di adrenalina e di cortisolo (2), mentre nelle donne potrebbe aumentare le concentrazioni di ossitocina (3).

A livello del SNC il rumore causa modificazioni del tracciato elettroencefalografico, e nei lavoratori esposti può provocare riduzione dell'attenzione, calo dell'efficienza ed aumento del carico di lavoro, turbe del sonno e dell'affettività, riduzione della memoria specie nei pazienti anziani, comparsa di sindromi ansiose ed ansioso-depressive, aumento dell'aggressività forse, come è stato dimostrato in animali da esperimento, anche attraverso una modificazione delle concentrazioni ippocampali dell'inibitore del diazepam (4-7). Interessante appare anche la possibilità che il rumore possa interferire con lo stato immunitario. Nell'uomo è stato infatti dimostrato che l'esposizione ad un rumore acuto influenza la capacità di proliferazione dei linfociti alla concanavalina e la percentuale di monociti (8).

Gli studi più interessanti ed approfonditi esistenti in letteratura riguardano tuttavia gli effetti del rumore sul sistema cardiovascolare. Sebbene infatti qualche Autore abbia cercato di ridimensionare le conseguenze dell'esposizione al rumore $(9,10)$, vari studi hanno dimostrato che l'esposizione al rumore è in grado di provocare modificazioni elettrocardiografiche (11), turbe della contrattilità cardiaca (12), aumenterebbe il rischio di cardiopatia ischemica (13), ed attraverso un'azione aritmogena potrebbe persino aumentare l'incidenza di morte improvvisa (14). Ormai certa è inoltre la possibilità che il rumore possa determinare un incremento della pressione arteriosa (15). Nei ratti è ad esempio sufficiente esporre gli individui con ipertensione border line al rumore per determinare un'ipertensione stabile (16), mentre in donne esposte a rumore cronico esiste una correlazione tra livello di rumore ed ipertensione (17). Eggsten ritiene addirittura possibile che il rumore possa essere 
direttamente responsabile di alterazioni strutturali dei vasi di resistenza (18), mentre secondo altri Autori il rumore sarebbe il terzo fattore causale dell'ipertensione dopo la familiarità ed il consumo di sale (19).

Non esistono invece in letteratura, studi che abbiano approfondito gli effetti del rumore sulla funzione renale, né, per quanto è a nostra conoscenza, è stata mai effettuata una adeguata valutazione dell'inquinamento ambientale in ambienti nosocomiali di tipo nefrologico, presupposto imprescindibile per una adeguata politica di prevenzione degli eventuali danni da rumore.

Scopo di questo studio è stato appunto quello di valutare l'entità del rumore in una Unità di terapia intensiva e dialisi, ed in particolare sia il livello sonoro ed il suo comportamento nelle 24 ore che le sue caratteristiche in funzione degli eventi che lo determinano, per verificare se esso possa effettivamente costituire un significativo momento di disturbo per il paziente nefropatico e per il personale del reparto.

\section{Materiali e metodi}

Lo studio è stato effettuato nei locali della nostra Unità di Terapia Intensiva e Dialisi (Fig. 1), le cui caratteristiche strutturali sono riassunte nella Tabella I.

Le misure sono state eseguite utilizzando un fonometro modulare Bruel \& Kiaer (mod. 2231) dotato di microfono (mod. 4155 ) e del modulo applicativo per la misura integrata del livello del rumore (mod. BZ7110). Ogni serie di misure veniva effettuata dopo un'accurata calibrazione dello strumento ottenuta sia mediante il generatore interno, sia mediante una sorgente standard (mod. 4230). Il tempo di misura è stato scelto pari a 60 secondi dal momento che non sono stati individuati o non sono stati considerati rumori con periodi maggiori di tale intervallo temporale, che corrisponde ad una frequenza di $0.017 \mathrm{~Hz}$. Per la ponderazione di frequenza, il fonometro BK2231 possiede al suo interno quattro filtri che permettono di eseguire le misure secondo standard diversi. Nel nostro caso è stata usata la curva ponderale A conforme alla normativa IEC 651. Le altre caratteristiche di funzionamento del fonometro sono riassunte nella Tabella II.

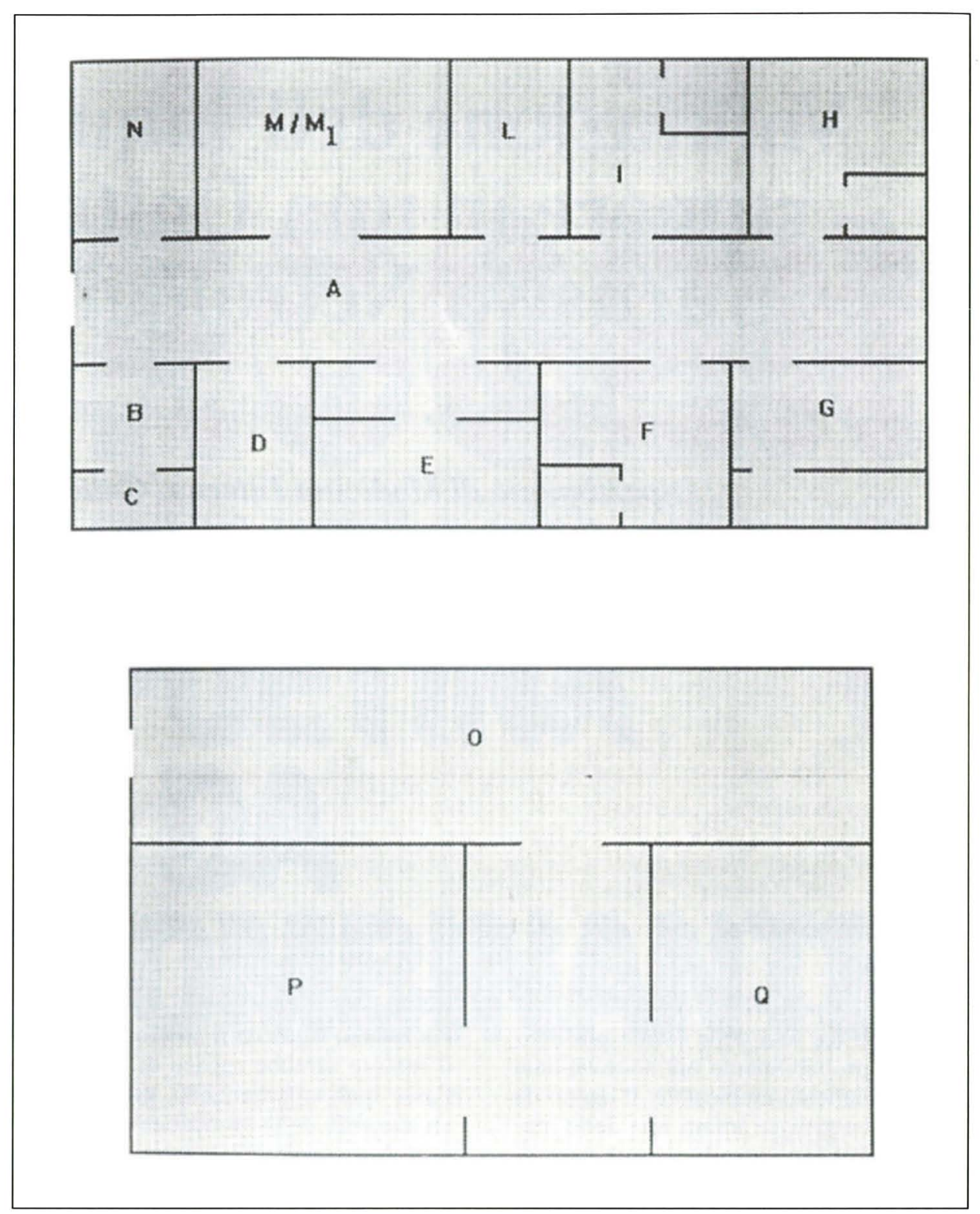

Fig. 1 - L'Unità di Terapia Intensiva (UTI) (piano superiore) e l'Ambulatorio di Nefrologia (piano inferiore)

I dati, durante l'acquisizione, venivano registrati nella memoria del fonometro e dopo elaborazione erano stampati su carta termica utilizzando la stampante grafica portatile mod. 2318 collegata al fonometro dal modulo di interfaccia mod. Z19101.

I risultati sono stati espressi come Livello Sonoro Equivalente (Leq). Tale livello rappresenta il livello di pressione sonora continuo stazionario, riferito ad un certo periodo di osservazione, corrispondente energeticamente a quello variabile che si verifica nello stesso intervallo di tempo.
Il Leq è infatti universalmente utilizzato come parametro di valutazione dell'inquinamento acustico di un ambiente.

Sono stati valutati tre diversi periodi in una giornata feriale (8-13 - 13-18 - 18$23)$, mentre è stato valutato il periodo 8 23 in una giornata festiva.

Le rilevazioni effettuate in sala dialisi sono state effettuate prima e dopo aver eseguito dei lavori di ristrutturazione che hanno consentito di migliorare le capacità fonoassorbenti dell'ambiente.

È stato inoltre effettuato un esame audiometrico di tutto il personale infermieri- 
TAB. I - CARATTERISTICHE DI ALCUNI DEI LOCALI DEL REPARTO UTI

\begin{tabular}{lcccc}
\hline Tipo di ambiente & $\begin{array}{c}\text { Cubatura } \\
(\mathrm{mc})\end{array}$ & $\begin{array}{c}\text { Superficie } \\
\text { fenestrata } \\
(\mathrm{mq})\end{array}$ & $\begin{array}{c}\text { Tipo di } \\
\text { pavimentazione }\end{array}$ & $\begin{array}{c}\text { Tipo di } \\
\text { rivestimento } \\
\text { alle pareti }\end{array}$ \\
\hline Corridoio & 107 & 130 & Marmo & Plastica \\
Medicheria & 64 & 3 & Marmo & Plastica \\
Stanza primario & 14 & 3 & Marmo & Plastica \\
Infermeria & 59 & 3 & Marmo & Plastica \\
Sala operatoria & 94 & 6 & Marmo & Plastica \\
Stanza degenti x4 & 111 & 3 & Marmo & Plastica \\
Stanza medici & 61 & 3 & Marmo & Plastica \\
Camera sterile & 63 & 4 & Marmo & Plastica \\
Stanza degenti x2 & 67 & 2 & Marmo & Plastica \\
Stanza steril. acqua & 65 & 2 & Marmo & Plastica \\
Sala Dialisi & 97 & 5 & Marmo & Plastica \\
Sala Dialisi ristrut. & 97 & 5 & Linoleum & Ceramica \\
Cucina & 30 & 2.8 & Marmo & Ceramica \\
\hline
\end{tabular}

TAB. II - CARATTERISTICHE DI MISURA DEL FONOMETRO

\begin{tabular}{lc}
\hline Parametro & Valore \\
\hline Correzione (Fattore K) & +0.6 \\
Correzione S.I. & FRONTAL \\
Risposta temporale & FAST \\
Fondo scala 1 (ambienti più rumorosi) & $40-110 \mathrm{~dB}$ \\
Fondo scala 2 (ambienti meno rumorosi) & $30-100 \mathrm{~dB}$ \\
\hline
\end{tabular}

stico ed ausiliario del reparto $(4 \mathrm{M}-4 \mathrm{~F}$; età media $32 \pm 5$ anni; durata della permanenza in reparto $5 \pm 2$ anni). Per tale scopo è stato utilizzato un audiometro Amplifon 300 ed un impedenziometro Amplifon 702. Lo studio è stato effettuato dopo consenso informato, al mattino, dopo almeno 24 ore di assenza dal reparto.

\section{Risultati}

La rilevazione fonometrica dei dati (Tab. III) ha evidenziato, come era ovvio aspettarsi, maggiori valori del Leq nelle ore diurne del giorno festivo, mentre il rumore tende ad attenuarsi nel corso della sera e durante la notte, con uno scarto massimo minimo di circa il 33\% (Tab. III). Il valore di picco massimo è stato quello registrato nella sala di medicheria nella mattina del giorno feriale (76.8 $\mathrm{dB}$ ), mentre nello stesso periodo il valore minimo è stato quello registrato nella sala di dialisi dopo i lavori di ristrutturazione $(43.7 \mathrm{~dB})$. Tale valore, così come quello registrato prima di tali lavori $(67.0 \mathrm{~dB})$, tiene conto delle macchine in funzione per l'emodialisi con 4 pazienti trattati, e la presenza del personale medi-

TAB. III - VALORI MEDI DEL LEQ RILEVATI NELL'ARCO DELLA GIORNATA NEI DIVERSI LOCALI DELL'UTI

\begin{tabular}{lcccccc}
\hline Postazione & & $\begin{array}{c}8-13 \\
\text { feriale }\end{array}$ & $\begin{array}{c}13-18 \\
\text { feriale } \\
\text { Leq dB (A) }\end{array}$ & $\begin{array}{c}18-23 \\
\text { feriale }\end{array}$ & $\begin{array}{c}8-23 \\
\text { festivo }\end{array}$ & $\begin{array}{c}\text { Valori } \\
\text { medi max } \\
\text { consigliati* }\end{array}$ \\
\hline Esterno & $\mathrm{X}$ & 70.4 & 76.2 & 55.7 & 60.5 & 50 \\
Interno & $\mathrm{Y}$ & 58.9 & 63.6 & 46.6 & 41.8 & $45-50$ \\
(Esterno UTI) & $\mathrm{A}$ & 66.8 & 48.9 & 39.8 & 47.3 & $45-50$ \\
Corridoio & $\mathrm{B}$ & 76.8 & 49.5 & 49.4 & 52.7 & $35-45$ \\
Medicheria & $\mathrm{C}$ & 66.6 & 49.6 & 42.0 & 53.7 & - \\
Stanza primario & $\mathrm{D}$ & 61.4 & 48.1 & 36.4 & 40.4 & $40-50$ \\
Infermeria & $\mathrm{E}$ & 48.8 & 41.4 & 37.3 & 41.2 & $35-45$ \\
Sala operatoria & $\mathrm{F}$ & 57.3 & 51.6 & 41.0 & 54.1 & $30-40$ \\
Stanza degenti & $\mathrm{G}$ & 59.9 & 49.6 & 40.3 & 45.7 & - \\
Stanza medici & $\mathrm{H}$ & 54.8 & 46.8 & 38.9 & 51.1 & $30-40$ \\
Stanza sterile & $\mathrm{I}$ & 65.3 & 45.8 & 38.8 & 53.9 & $30-40$ \\
Stanza degenti & $\mathrm{L} 2$ & 63.8 & 57.4 & 55.7 & 54.4 & - \\
Stanza ster. acqua & $\mathrm{M}$ & 67.0 & 47.2 & 38.8 & 40.5 & - \\
Sala Dialisi & $\mathrm{M} 1$ & 43.7 & 38.0 & 33.0 & 36.8 & - \\
Sala Dial. ristrut. & $\mathrm{N}$ & 67.5 & 49.2 & 38.7 & 49.7 & - \\
Cucina & $\mathrm{O}$ & 66.8 & 57.5 & 38.0 & 39.2 & $50-55$ \\
Ambulatorio & $\mathrm{P}$ & 61.5 & 49.5 & 51.9 & 45.3 & $40-50$ \\
Laboratorio & $\mathrm{Q}$ & 61.8 & 61.3 & 58.9 & 59.0 & $50-55$ \\
Stanza servizi & $\mathrm{R}$ & 68.7 & 65.3 & 42.0 & 39.9 & $40-50$ \\
Stanza attesa & & & &
\end{tabular}


TAB. IV - VARIAZIONE DEI LIVELLI NORMALI DI RUMORE DURANTE EVENTI OCCASIONALI

\begin{tabular}{llcc}
\hline Tipi di eventi & Luogo & $\begin{array}{c}\text { Media del } \\
\text { valore } \\
\text { Leq (A) dB }\end{array}$ & $\begin{array}{c}\text { Valore } \\
\text { occasionale } \\
\text { Leq (A) dB }\end{array}$ \\
\hline Visite dei pazienti & Stanza con 4 pazienti (18.00-23.00) & 41.0 & 61.0 \\
Campanelli telefonici & Medicheria (8.00-13.00) & 64.8 & 68.7 \\
Campanello & Medicheria (8.00-13.00) & 64.8 & 76.8 \\
Emogasanalisi & Medicheria (18.00-23.00) & 49.4 & 54.1 \\
Centrifuga & Medicheria (18.00-23.00) & 74.3 & 67.4 \\
Frigorifero & Medicheria (18.00-23.00) & 49.4 & 51.3 \\
\hline
\end{tabular}

TAB. V - RIDUZIONE DEL RUMORE GENERATO DA MACCHINE DIVERSE IN AMBIENTI DIFFERENZIATI PER CARATTERISTICHE INSONORIZZANTI

\begin{tabular}{lccc}
\hline Ambiente & Sifra 907 & Hospal AFB & Gambro AK 10 \\
\hline Sala Dialisi & 62.8 & 61.7 & 67.7 \\
Sala Dialisi ristrutt. & 52.9 & 48.4 & 53.6 \\
\hline
\end{tabular}

co (due) e paramedico (tre).

Nel periodo diurno del giorno feriale esaminato l'entità del rumore è stata sempre superiore ai valori medi consigliati dal DPCM 01.03.1991 (GU 8.03.1991), con l'unica eccezione della sala di dialisi dopo i lavori di ristrutturazione.

I valori rilevati subivano ulteriori importanti incrementi durante eventi occasionali (Tab. IV).

L'uso delle varie macchine di dialisi comportava un inquinamento sonoro nettamente diverso prima e dopo le modificazioni apportate alla sala (Tab. V).

Le misurazioni ottenute possono essere considerate attendibili. Secondo una prassi consolidata le misurazioni sono state infatti ripetute in un giorno diverso, e lo scarto risultante è stato compreso tra 1 ' $1.3 \%$ ed il $2.3 \%$.

Per ciò che riguarda la valutazione dei disturbi uditivi del personale di reparto, è stata rilevata una modesta ipoacusia per le alte frequenze in due degli otto soggetti esaminati.

\section{Discussione}

Sia pure in assenza di dati sperimentali specifici sugli effetti del rumore sulla funzionalità renale, è indubbio che anche soltanto le modificazioni cardiovascolari indotte dal rumore quali l'ipersimpaticotonia con vasocostrizione periferica precapillare ed aumento delle resistenze periferiche, la riduzione della gittata cardiaca, e soprattutto l'incremento pressorio, non possono non rivestire un aspetto negativo per il paziente nefrologico.

In ogni caso in un paziente cronico quale è il paziente dializzato, lo stress da rumore costituisce un logorante fattore aggiuntivo di tensione capace di aggravare il già precario equilibrio psichico e motivazionale del paziente, e di rendere ancora più difficile l'accettazione dello stato di malattia ed il rapporto di dipendenza dall'unità di dialisi. Per quanto riguarda gli effetti del rumore sull'udito dei parasanitari presenti nel reparto, essi non sembrano particolarmente rilevanti, specie se confrontati con i valori di riferimento per sesso e fascia di età (20). È inoltre da tenere presente che tutti i soggetti erano in servizio da meno di 5 anni. Sebbene un danno immediato dell'udito si abbia per rumori di intensità superiore a $120 \mathrm{~dB}$, il rischio di danno acustico può avere inizio a livelli di molto inferiori, se l'esposizione del soggetto alla sorgente sonora è molto prolungata. Un rumore di soli $85 \mathrm{~dB}$ sopportato per 40 anni provoca una perdita di udito di circa 30 dB nel 35\% dei soggetti. Per tale motivo ad esempio il Committe on Hygiene Standards raccomanda che la quantità di energia sonora non superi i $105 \mathrm{~dB}$, corrispondenti ad un livello equivalente continuo di $90 \mathrm{~dB}(\mathrm{~A})$ per 30 anni lavorativi di 48 settimane, per 40 ore settimanali.

Sebbene l'intensità del rumore nel reparto UTI da noi valutato si mantenga dentro tali valori è da considerare che il danno acustico non costituisce l'unico elemento di rischio per il personale del reparto.

Il lavoro del personale sanitario in una unità di degenza quale la UTI differisce probabilmente da quello di un normale reparto ospedaliero. Il carico di responsabilità che l'esecuzione di quasi ogni direttiva comporta, rende di estrema importanza l'esistenza di una comunicazione verbale chiara ed inequivocabile (21). La presenza di rumore determina probabilmente un ulteriore carico di stress per il personale parasanitario, con possibile comparsa di pericolosi cali di attenzione e di efficienza.

Nell'Arbetsstatten-Verordnung del marzo 1975 e nella raccomandazione VD12058 Blatt 3 dell'aprile 1981 della Germania Occidentale viene infatti prescritto che nei locali di medicazione e di controllo medico in ospedali, policlinici e ambulatori con limitata sollecitazione psichica il leq (dBA) non debba superare il valore massimo consentito di 55 (con un valore raccomandato di 50 ), ed in quelli con notevole sollecitazione psichica (quali appunto le UTI) il valore massimo di 40 con valore raccomandato di 35 leq (dBA) (22). Tali valori risultano invece ampiamente superati persino dopo i lavori di ristrutturazione eseguiti per migliorare l'isolamento acustico della sala di dialisi.

A quest'ultimo proposito è importante sottolineare come una prevenzione ambientale adeguata del danno da rumore debba in effetti essere eseguita già nella fase di progettazione dell'unità intensiva. L'adeguamento delle strutture, come è avvenuto nel nostro caso, non garantisce infatti i medesimi risultati, e determina un ovvio incremento dei costi. 
Una razionale pianificazione degli ambienti permette infatti di ovviare a problemi altrimenti insolubili successivamente, quali la realizzazione degli edifici nelle vicinanze di sorgenti di rumore esterne insopprimibili quali aeroporti, autostrade, industrie. In particolare poi l'esecuzione preventiva di una mappa del rumore può essere utile per dislocare razionalmente le diverse sorgenti di rumore e posizionare opportunamente quelle a più forte rumore allontanandole dai luoghi di degenza e terapia. In sede di progettazione è inoltre facile intervenire sulla propagazione del rumore con l'uso di coperture integrali o parziali delle macchine più rumorose (sistemi di aerazione e riscaldamento, lavatrici, ecc), l'impiego di soffitti alti e non a cupola, di pavimenti in gomma, di spioncini alle porte per evitare aperture inutili, una scelta adeguata delle ruote di tutte le apparecchiature mobili, e soprattutto l'uso di materiale fonoassorbente per il rivestimento delle pareti. Solo quest'ultimo accorgimento è infatti in grado di determinare di per sé una riduzione del livello sonoro di circa 7-8 dB rispetto ad ambienti con pareti fonoriflettenti $(23,24)$.

Alla luce di quanto detto può apparire strano che non esistano in Italia studi adeguati sull'inquinamento da rumore in ambiente nosocomiale, o che non venga ad essi dato il giusto risalto. $\grave{E}$ possibile che la difficoltà ad eseguire indagini per la verifica della salubrità sonora negli ambienti di lavoro dipenda dal timore dell'eventuale necessità di effettuare lavori di bonifica e dell'avvio di interventi di carattere ispettivo.

A nostro avviso tuttavia, il definitivo riconoscimento del rumore come importante agente patogeno, rende indilazionabile uno studio approfondito sull'argomento che consenta di migliorare il confort lavorativo del personale impiegato nei reparti, e soprattutto garantisca in modo assoluto la tutela del paziente.

\section{Riassunto}

Utilizzando un fonometro ad alta sensibilità sono stati valutati i livelli di rumore nei diversi ambienti di una Unità di Terapia Intensiva e Dialisi prima e dopo l'esecuzione di lavori tesi a mi- gliorare l'insonorizzazione delle stanze. I livelli di rumore registrati nelle ore diurne dei giorni feriali sono risultati sempre superiori a quelli raccomandati per i luoghi di degenza e terapia.

Gli Autori hanno analizzato gli aspetti negativi che una condizione di inquinamento sonoro riveste per il paziente nefropatico e per il personale sanitario del reparto.

\section{BIBLIOGRAFIA}

1.

Maugeri S, Odescalchi CP. Il rumore industriale. Problemi attuali, patologia, prevenzione. Securitas 1968; 53: 33-8.

2. Agnes F, Sartorelli P, Abdi BH, Locatelli A. Effect of transport loading or noise on blood biochemical variables in calves. Am $\mathrm{J}$ Vet Res 1990; 51: 1679-81.

3. Sanders G, Freilicher J, Lightman SL. Psychological stress of exposure to uncontrollable noise increases plasma oxytocin in high emotionality women. Psychoneuroendocrinology 1990; 15: 47-58.

4. Suter AH. Noise and its effects. In: Shapiro S.A. The Dormant Noise Control Act and options to abate noise pollution. Washington: Administrative Conference of the United States, 1991.

5. Belojevic G. Subjective sensitivity to noise. Srp Arch Celok Lek 1991; 119: 221-3.

6. Molander B, Backman L. Age differences in the effects of background noise on motor and memory performance in a precision sport. Exp Aging Res 1990; 16: 55-60.

7. Ferrarese C, Mennini T, Pecora $\mathrm{N}$, et al. Acute noise stress in rats increases the levels of diazepambinding inhibitor (DBI) in hippocampus and adrenal gland. Psycopharmacology 1991; 103: 339-42.

8. Weisse CS, Pato CN, McAllister CS, Littman R, Breier A, Paul SM. Differential effects of controllable and uncontrollable acute stress on lymphocyte proliferation and leukocyte percentages in humans. Brain Behav Immun 1990; 4: 339-51.

9. Colletti V, Fiorino FG. Myocardial activity during noise exposure. Acta Otolaryngol (Stockh) 1987; 104: 217-24.

10. Dudek B, Marszal-Wie- 
sniewka M, Merecz-Kot D, Sulkowki W, Bortkiewicz A. Effects of noise on cognitive processes of individuals in a laboratory experiment. Pol J Occup Med 1991; 4: 269-79.

11. Tomei F, Tomao E, Papaleo B, Baccolo TP, Alfi P. Study of some cardiovascular parameters after chronic exposure to noise. Int J Cardiol 1991; 33: 393-9.

12. Miller MB. Effect of working conditions on myocardial contractility in tube-rolling mill worker. Gig Tr Prof Zabol 1991; 3: 10-2.

13. Idzior-Walus B. Coronary risk factors in men occupationally exposed to vibration and noise. Eur Heart J 1987; 8: 1040-6.

14. Topaz O, Castellanos A, Grobman LR, Myerburg RJ. The role of arrhythmogenic auditory stimuli in sudden cardiac death. Am Hearth J 1988; 116: 222-6.

15. Theorell T. Family history of hypertension, an individual trait interacting with spontaneously occurring job stressors. Scand J Work Environ Health 1990; 16: (S1) 74-9.

16. Gamallo A, Alario P, Villanua MA, Nava MP. Effect of chronic stress in the blood presure in the rat: ACTH administration Horm Metab Res 1988; 20: 336-8.

17. Green MS, Scharwtz K, Harari G, Najenson T. Industrial noise exposure and ambulatory blood pressure and heart rate. J Occup Med 1991; 33: 879-83.

18. Eggersten R, Svensson A, Magnusson M, Andren L. Hemodynamic effects of loud noise before and after central sympathetic nervous stimulation. Acta Med Scand 1987; 221: $159-64$.

19. Zhao YM, Zhang SZ, Selvin S, Spear RC. A dose response relation for noise induced hypertension. $\mathrm{Br} \mathbf{J}$ Ind Med 1991; 48: 179-84.

20. Regione Emilia Romagna USL 14. Valori di riferimento per il calcolo della soglia uditiva attesa di maschie femmine per anno di età. Contributi. 1993; 34: 1-83.

21. Cosa M, Barbaro S, Guffaldi D, Cosa G. Effetti del rumore sulla trasmissione e sulla comprensione della voce parlata. Effetti sulla qualità e sulla durata del sonno. Difesa sociale 1993; 5: 18-22.

22. Ianaccone A, Castagnoli A. Misura e valutazione della rumorosità negli uffici e nei locali dove viene svolta un'attività intellettuale. Atti IV Convengo Ecologico Europeo Inquinamento da rumore. Ed. Sfameni pag 273-85. Taormina 26-8 Giugno 1981.

23. Cosa M. Il rumore all'interno degli edifici. In: Il rumore urbano ed industriale. Ed. Roma 1980; 259323.

24. Alzati A. Acustica ambientale ed insonorizzazione. Etos libri 1984; 103-5. 